\title{
SHED ameliorated concanavalin A-induced autoimmune hepatitis by protecting hepatocytes from apoptosis
}

\section{Yikun Zhou}

Peking University School of Stomatology

Ruili Yang ( $\sim$ ruiliyangabc@163.com )

Peking University School of Stomatology https://orcid.org/0000-0002-3283-9893

\section{Lingsu Zhu}

Zhengzhou University First Affiliated Hospital

\section{Huaming Huang}

Peking University School of Stomatology

\section{Shengjie Cui}

Peking University School of Stomatology

\section{Ting Zhang}

Peking University School of Stomatology

\section{Yanheng Zhou}

Peking University School of Stomatology

\section{Research}

Keywords: autoimmune hepatitis, stem cells from human exfoliated deciduous teeth, concanavalin A, apoptosis, NF-KB

Posted Date: March 23rd, 2020

DOI: https://doi.org/10.21203/rs.3.rs-17847/v1

License: (a) (1) This work is licensed under a Creative Commons Attribution 4.0 International License. Read Full License 


\section{Abstract}

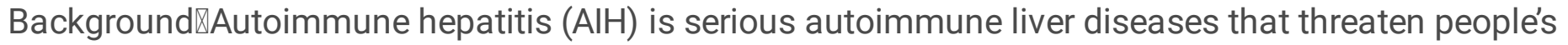
health worldwide, emphasizing the need to identify novel treatment. Stem cells from human exfoliated deciduous teeth (SHED), which is easy to obtain and non-invasive, showed pronounced proliferation and immunomodulation capacity. This study aims to investigate the effect of SHED on ConA-induced AlH and the potential underlying mechanisms.

Methods: We used a concanavalin A (ConA) induced acute hepatitis mouse model and in vitro co-culture system to study the protective effects of SHED on ConA-induced autoimmune hepatitis and the underlying mechanisms.

Results: SHED infusion could prevent aberrant histopathological architecture of liver with infiltration of abundant of $\mathrm{CD} 3+, \mathrm{CD} 4+, \mathrm{TNF}-\mathrm{a}+$ and IFN- -+ inflammatory cells induced by ConA. The expression of ALT and AST which indicated the liver function significantly elevated in hepatitis mice. While SHED infusion could block the elevation of ALT and AST induced by ConA. Mechanistically, Con-A upregulated TNF-a and IFN-y expression activated NF-KB pathways to induced hepatocyte apoptosis, resulting in acute liver injury. SHED administration protected hepatocytes from Con-A-induced apoptosis.

Conclusions: These results demonstrated that SHED alleviated ConA-induced acute liver injury via inhibition of hepatocyte apoptosis mediated by the NF-KB pathways. Our findings could provide a potential prevention and therapeutic strategy for hepatitis and acute hepatic injury.

\section{Introduction}

Autoimmune hepatitis $(\mathrm{AlH})$ is an immune-mediated liver inflammatory disease that has a variety of clinical manifestations, including occult onset without any symptoms, acute onset and even acute liver failure[1]. The course of AlH can undulate, which may start from acute hepatitis, leading to fibrosis, cirrhosis, liver transplantation, and even death in a short period of time[2,3]. The therapeutic aim of autoimmune hepatitis is to alleviate the state of liver inflammation and prevent fibrosis. Generally, the treatment of $\mathrm{AlH}$ requires long-term or even lifelong use of immunosuppressants, which may cause serious side effect. $10 \%-20 \%$ of $\mathrm{AlH}$ patients are not sensitive to immunosuppressant treatment and can't be well controlled even after treatment, who will develop to end-stage cirrhosis[4, 5]. AlH is becoming a worldwide health problem, which highlights the need to explore alternative treatment strategy.

As mesenchymal stem cells (MSCs) have multi-lineage differentiation and immunomodulation properties, stem cells therapy for inflammation related diseases have attracted more attention recently. It has reported that MSC infusion could be alleviate a variety of diseases such as autoimmune disease, nerve injury, spinal cord injury and diabetes[6]. Previous studies have shown that injection of bone marrow mesenchymal stem cells (BMMSCs), adipose mesenchymal stem cells and tonsil derived stem cells can alleviate the autoimmune hepatitis induced by ConA in mice [7-9]. However, the limited sources of BMMSCs have greatly narrowed their therapeutic applications. Stem cells from human exfoliated 
deciduous teeth (SHED) was found in the pulp of deciduous teeth, which showed more pronounced proliferation and immunomodulation capacity compared to BMMSCs[10, 11]. Yamaza also reported that the spleen transplantation of SHED significantly improved CCl4 induced cirrhosis, inflammation and fibrosis of rats[12]. SHED was also reported to differentiate into hepatocytes in vitro,indicating that SHED maybe the proper stem cells for treat hepatitis[13]. In view of the above effects of SHED, we wonder whether the non-traumatically collected stem cells-SHED can block the occurrence of autoimmune hepatitis.

Concanavalin A (ConA), from the jack bean Canavalia ensiformis, could bound to sugar residues on the surface of multiple cell types[14, 15]. It has been reported that AlH induced by ConA has the same pathogenicity and histopathological characteristics in accordance with AlH. ConA can induce hepatocyte damage by activating inflammation cytokines produced by macrophages and $T$ cells. It has reported that the inflammatory cytokines such as interleukin-2 (IL-2),IL-6ロtumor necrosis alpha (TNF-a) and interferon gamma (IFN- $\gamma$ ) play important role during the process of acute hepatitis[16-18]. Therefore, the previous studies used drugs to down regulate the relevant pro-inflammatory factors through different channels (TRADD/TRAF2 JAK2/STAT, ROS, PI3K and JNK), so as to achieve the purpose of reducing liver damage and treating $\mathrm{AlH}[19-21]$. However, the effect of these drugs is limited in some phenomena, and its exact mechanism needs more studies. SHED have showed profound immunomodulatory ability to inhibit T lymphocyte proliferation and anti-inflammation in vitro and in vivo[22]. Whether SHED infusion could block the acute inflammation caused by ConA need to be further investigated.

Here, we explored the effects of SHED on ConA-induced AlH in mice, as well as the potential underlying mechanisms. The results showed that SHED infusion alleviated ConA-induced acute liver injury via inhibition of hepatocyte apoptosis mediated by the NF-KB pathways.

\section{Materials And Methods}

\section{Animals and treatment}

Male Balb/c mice (6-8 weeks old, $23 \pm 2 \mathrm{~g}$ ) were purchased from Vital River Laboratory Animal Technology (Beijing Vital River Laboratory Animal Technology, China). Mice were randomly assigned to three groups: control group $(n=10)$; ConA group $(n=10)$; intravenous injections with $20 \mathrm{mg} / \mathrm{kg}$ ConA (20 $\mathrm{mg} / \mathrm{kg}$ weight, Sigma, USA); SHED+ConA group $(\mathrm{n}=10)$; intravenous injections with SHED cell $\left(1 \times 10^{6}\right) 7 \mathrm{~d}$ prior to ConA challenge. After injection of ConA for $24 \mathrm{~h}$, the mice were scarified for further analysis (Fig.1a). The protocols were approved by the Animal Care and Use Committee of the Health Science Center, Peking University (\# 2015-186).

\section{Biochemical assay}

Mice serum was collected for the inflammatory cytokine, ALT and AST analysis. The levels of ALT and AST were evaluated by the transaminase kit according to the manufacturer's instructions (Nanjing 
Jiancheng Bioengineering Institute, Nanjing, China). TNF-a and IFN-y levels were measured using ELISA kits according to the manufacturer's instructions》ebioscience, USA囚[19].

\section{Immunohistochemistry}

For immunohistochemistry, the sections were dewaxed with xylene, treated with gradient concentration of ethanol, washed with deionized water, followed by heated with a microwave in $10 \mathrm{mmol} / \mathrm{L}$ sodium citrate (PH 6), added with $0.05 \%$ Tween 20 , then blocked for 1 hour with $5 \%$ BSA and $0.1 \%$ Triton X-100 in PBS. Anti-CD3 (1:100), anti-CD4(1:100), anti-total caspase3 (1:100), anti TNF-a (1:100), anti IFN-ץ (1:100) and anti NF-KB (1:400) were incubated overnight at $4{ }^{\circ} \mathrm{C}$. After washed three times, The sections were incubated with secondary antibody at room temperature for $1 \mathrm{~h}$, and observed under microscope (Leica, Wetzlar, Germany). The positive area was measured with Image-Pro Plus software 6.0 (Media Cybernetics, Silver Spring, MD, USA).

\section{Immunofluorescence}

After washed in PBS for 5 min, the frozen sections of tissues were treated with $0.1 \%$ Triton to rupture the membranes at room temperature for $30 \mathrm{~min}$, and non-specific antigen binding site was blocked by $5 \%$ BSA. Rabbit anti-total caspase3 (1:100), anti-pNF-KB (1:400) and anti-NF-KB (1:400) were incubated overnight at $4{ }^{\circ} \mathrm{C}$. Nuclear staining was performed by DAPI (Vector Lab, Burlingame, CA)mounting medium after incubated with secondary antibody for $1 \mathrm{~h}$. All sections were observed with Zeiss fluorescence microscope (Zeiss, Thornwood, NY).

\section{Cell culture and treatment}

Murine liver cell line NCTC-1469 cells were purchased from the Institute of Biochemistry and Cell Biology, Chinese Academy of Science (Shanghai, China). Stem cells from human exfoliated deciduous teeth (SHED) cells were provided by Oral stem cell bank (Beijing, China) and isolated as previously reported[10]. NCTC-1469 cells were cultured in DMEM supplemented with $10 \%$ fetal bovine serum (FBS), $100 \mathrm{U} / \mathrm{mL}$ penicillin, and $100 \mathrm{U} / \mathrm{mL}$ streptomycin. SHED cells were cultured in a-MEM supplemented with $15 \%$ fetal bovine serum (FBS), $100 \mathrm{U} / \mathrm{mL}$ penicillin, and $100 \mathrm{U} / \mathrm{mL}$ streptomycin. NCTC cells $\left(2 \times 10^{5}\right.$ cells/well, 2 $\mathrm{mL} /$ well) were cultivated in 6-well plates and incubated with ConA of different concentrations $(10 \otimes 20 \rrbracket 50 \rrbracket$ $80 \otimes 100 \mu \mathrm{g} / \mathrm{ml}$ ), which were studied in cell proliferation by cell counting. Then we selected the appropriate treatment concentration $(50 \mu \mathrm{g} / \mathrm{ml})$. In order to study whether SHED cells can inhibit the death of NCTC cells caused by ConA in vitro. PKH26 labeled NCTC-1469 cells were inoculated into 6-well plates with density of $2 \times 10^{5}$ cells $/ \mathrm{mL}$ for $12 \mathrm{~h}$. Then the cells were cultured with $50 \mu \mathrm{g} / \mathrm{ml}$ Con A and SHED cells $(1 \times$ $10^{5}$ cells/well) for $24 \mathrm{~h}$. The cells on the slide were fixed and observed with Zeiss fluorescence microscope (Zeiss, Thornwood, NY).

\section{Determination of apoptosis and DNA damage}


Apoptosis in liver tissues was detected by Terminal dUTP nick-end labeling (TUNEL) staining. The paraffin-embedded sections were deparaffinized and digested with $20 \mu \mathrm{g} / \mathrm{mL}$ proteinase $\mathrm{K}$ for $30 \mathrm{~min}$ at room temperature. After washed with PBS, the slices were incubated with the TUNEL reaction overnight at $4^{\circ} \mathrm{C}$, followed by incubation with rabbit anti-mouse fluorescein isothiocyanate, and DAPI for nuclear labeling. The TUNEL ${ }^{+}$cells were observed and counted in at least three different fields per section.

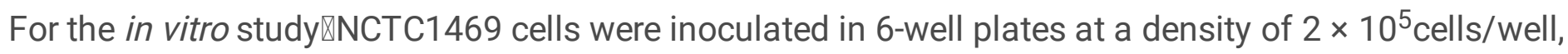
followed by the addition of ConA $(50 \mu \mathrm{g} / \mathrm{ml})$ and SHED cell $\left(1 \times 10^{5}\right.$ cells/well). After 24 hours of culture, the cells were collected, washed with PBS, and stained with Annexin V apoptosis detection kit. Flow cytometry was used to observe the fluorescence of cell. The cells on the slide were fixed and then immunofluorescence staining was used to determine the expression of apoptosis. After incubation in blocking buffer, cells were incubated with anti-caspase $3(1: 400)$ overnight at $4^{\circ} \mathrm{C}$, followed by incubation with goat anti-rabbit fluorescein isothiocyanate. Nuclear staining was performed by DAPI. The sections were observed with Zeiss fluorescence microscope (Zeiss, Thornwood, NY).

\section{Result}

\section{SHED moderated ConA-induced acute liver injury in mice}

To analyze the effects of SHED infusion to autoimmune hepatitis induced by Con A, we infused the SHED cells $\left(1 \times 10^{6}\right) 7$ days prior to Con A injection (Fig.1a). The results showed that pretreatment of SHED infusion significantly inhibited transaminase (ALT and AST) release of AlH (Fig.1b,1c). Anatomical and histological examinations, which were used to estimate the liver injury, showed that the structure of liver lobule and the arrangement were aberrant with inflammatory cells infiltration and massive necrotic areas in the hepatitis group. Infusion of SHED could significantly restore the structure of liver and reduce inflammatory cells infiltration (Fig.1d,1e). The results showed that SHED infusion demonstrated protective effect on acute liver injury.

\section{SHED-pretreatment reduced inflammation in ConA-induced hepatitis}

T cell mediated immune response plays a crucial role in the occurrence and development of autoimmune hepatitis. We further explored the underlying mechanism by analyzing the $T$ cells infiltration and they mediated the inflammation. The results showed that the number of $\mathrm{CD}^{+}$and $\mathrm{CD} 4^{+} \mathrm{T}$ cells in the liver tissues of mice in ConA group was significantly higher than that in control group, and that SHED pretreatment was markedly reduced the number of $\mathrm{CD} 3^{+}$and $\mathrm{CD} 4^{+} \mathrm{T}$ cells (Fig.2a-2c). Hepatitis is associated with changes in the levels of inflammatory cytokines. TNF-a and IFN- $\gamma$ were significantly increased in the ConA-treated group, while pretreatment with SHED cells significantly reduced the ConAinduced increase of TNF- $a$ and IFN-y expression in liver tissues (Fig.2d-2f). In addition, biochemical assay was used to detect the level of TNF-a and IFN-y in mouse serum, which are consistent with the results of in the liver tissues (Fig.2g,2h). These results showed that SHED played an immunomodulatory role in AlH mice, which alleviated liver tissue damage by inhibiting the activation of Th1 cell mediated inflammation. 


\section{SHED-pretreatment inhibited apoptosis of hepatocytes induced by Con A}

As ConA could induce hepatocyte damage, we analyzed whether ConA treatment affected the liver cells apoptosis. The results showed that ConA group showed more apoptosis of liver cells, and SHED significantly reduced the trend of apoptosis (Fig.3a, 3b). In order to test whether SHED infusion could protect liver cells from apoptosis, we used mouse liver cells NCTC 1469 co-cultured with SHED in vitro. ConA treatment decreased the viability of NCTC cells in a dose-dependently manner (Figure S1a, S1b). NCTC cells were stimulated with ConA at concentration of $50 \mu \mathrm{g} / \mathrm{mL}$ for $24 \mathrm{~h}$, then co-cultured with SHED to analyze the protective effect of SHED. The data showed that pretreatment with SHED considerably increased the viability of NCTC cells which inhibited by ConA treatment (Figure S1c, S1d). To verify the effects of SHED, NCTC cells were labeled with PKH26 and co-cultured with SHED, the results showed that PKH26 labeled NCTC cells significantly decreased after ConA treatment, while SHED co-culture could block the decrease of live PKH26 labeled NCTC cells (Fig.3c,3d). Furthermore, flow cytometry showed ConA treatment induced a remarkable (Annexin- $\mathrm{V}^{+} \mathrm{PI}^{-}$and Annexin $\left.-\mathrm{V}^{+} \mathrm{PI}^{+}\right)$cell apoptosis, which could be blocked by SHED pretreatment (Fig.3e,3f,3g). Caspase3 is one of the most important end shear enzymes in the process of apoptosis, we found that the expression of Caspase 3 were upregulated in ConA treated NCTC cells, while SHED co-culture decreased the up-regulated expression of caspase3 by ConA treatment, as assessed by immunofluorescence staining (Fig.4a,4b). Furthermore, we found the number of caspase $3^{+}$cells were also increased in the liver of ConA induced-hepatitis mice. SHED pretreatment could block the increase of caspase $3^{+}$cells in liver. (Fig.4c,4d). These results indicated that SHED infusion could protect hepatocytes from apoptosis induced by ConA.

\section{SHED infusion inhibited the NF-KB signaling pathway in ConA-induced hepatitis}

The inflammatory cytokines TNF- $a$ and IFN- $\gamma$ were elevated in ConA induced hepatitis, as NF-KB pathway played crucial in these cytokine mediated inflammation[23]. We analyzed the NF-kB pathway and found that the number of NF-KB (Fig5a,5b) and p-NF-KB (Fig5c,5d) positive cells were significantly increased in the ConA-treated group and significantly decreased in the SHED cell-pretreated groups. In addition, the results of immunohistochemical staining were consistent with immunofluorescence staining (Fig5e,5f). It suggested that SHED cells treatment might ameliorate liver injury in ConA-induced hepatitis in partly through the NF-KB pathway.

\section{Discussion}

Autoimmune hepatitis (AlH) is an immune-mediated inflammatory disease of the liver with increasing incidence and mortality rate, which can exist in acute or chronic forms. It is characterized by the elevation of serum transaminase and immunoglobulin $\mathrm{G}$ levels and positive circulating autoantibody [24]. Here we found that pretreatment with SHED 7 days showed protective effects for ConA induced hepatitis to restore the liver structure and reduce the level of serum transaminase. Studies have reported several MSCs could partially alleviate the hepatitis induced by ConA in mice when infusion MSCs with ConA at the same time[8, 9]. Here, we firstly reported that pretreatment with SHED group showed protective effects 
for acute hepatitis. As ConA induced hepatitis progressed rapidly, it caused more than $50 \%$ mice death without intervention in 24 hours (data was not shown). The phenomenon suggests that the earlier infusion of SHED may benefit for the treatment of acute hepatitis using stem cells. SHED is derived from the pulp of deciduous teeth, which is easy to obtain and non-invasive[25]. These data indicated the pretreatment with SHED maybe one alternative strategy to prevent and treat severe acute hepatitis and liver injury.

TNF- $\mathrm{a}$ and IFN- $\gamma$ secreted by T cells play important roles in inflammatory response[26]. Previous studies have shown that mice pretreated with TNF-a inhibitors or anti TNF- $a$ antibodies can reduce ConA induced hepatitis[27]. TNF- $\alpha$ and IFN- $\gamma$ knockout mice could not be induced liver damage after ConA stimulation[26-28]. It's reported that SHED possessed superior anti-inflammatory effects compared to BMMSCs[29]. In this study, the number of $\mathrm{CD}^{+}$and $\mathrm{CD} 4^{+} \mathrm{T}$ lymphocytes in the liver was decreased by injection of SHED, so as to inhibit the inflammatory factors produced by Th1 cells, and down regulate the expression of TNF- $a$ and IFN- $y$ in the liver tissues and peripheral blood. In vitro, we found that SHED can reverse the decrease of Con-induced liver cells, and then we studied its effect on the apoptosis of liver cells. The results indicated that SHED could reverse liver cells apoptosis induced by ConA. Several pathways, such as NF-KB, Fas/FasL,JNK, MAPK and ROS, play crucial roles in cell apoptosis[30-32]. Based on the significant protective effects of SHED, we explored the potential underlying mechanisms of SHED against ConA induced apoptosis. The results showed that pretreatment with SHED could effectively inhibit the phosphorylation of p65, suggesting that SHED might inhibit the activation of NF-KB by reducing the expression of TNF-a and IFN-y, thus protecting mice from the invasion of AlH [3235].Since apoptosis may also be regulated by death receptor or mitochondrial pathway, caspase family needs to be activated in both cases [36,37]. The results showed that SHED may block the activation of caspase3 to inhibit the external apoptosis (Fig. 6). However, the details of the mechanism and whether our signaling contributed to the protective effects of SHED to ConA induced hepatitis needs to be further investigated.

\section{Conclusions}

In summary, the results showed that the SHED exhibited protective effects in a ConA-induced mouse AlH model. SHED treatment could inhibit the activation of Th1 cells and reduce the secretion of TNF-a and IFN- $\gamma$ to inhibit the activation of NF-KB pathway induced by ConA, so as to reduce the apoptosis of hepatocytes and the inflammatory damage of liver. Our results suggested that SHED could be used as a potential preventing and therapeutic strategy for acute hepatitis and liver injury.

\section{Declarations}

\section{Availability of data and materials}

The datasets used and/or analyzed during the current study are available. 


\section{Abbreviations}

a-MEM®Minimal essential medium-alpha

AlH: Autoimmune hepatitis

ALT: Alanine aminotransferase

AST: Aspartate aminotransferase

BMMSCs: Bone marrow mesenchymal stem cells

CCl4: Carbon tetrachloride

ConA: Concanavalin A

DAPI:4',6-Diamidino-2-phenylindole

DMEM: Dulbecco's modified Eagle's medium

FasL: Fas ligand

FBS: Fetal bovine serum

IL: Interleukin

IFN-ץ: Interferon gamma

JAK: Janus-family tyrosine kinase

JNK: Jun N-terminal kinase

MAPK: Mitogen-activated protein kinase

MSCs: Mesenchymal stem cells

NCTC 1469 :normal liver cells

NF-кB: nuclear factor kappa B

PBS: Phosphate-buffered saline

PDLSCs: Periodontal ligament stem cells

PI3K: Phosphoinositide 3 kinase

ROS: Reactive oxygen species 
SHED: Stem cells from human exfoliated deciduous teeth

STAT: Signal transducer and activator of transcription

TNF-a: Tumor necrosis alpha

TRADD: TNF receptor-1-associated death domain protein

TRAF: TNF receptor-associated factor

TUNEL: Terminal dUTP nick-end labeling

\section{Acknowledgements}

Thank for the Oral Stem Cell Bank of China providing the SHED cells.

\section{Funding}

This work was supported by the National Natural Science Foundation of China No. 81970940 (R.Y), 81600865 (R.Y.), Beijing Natural Science Foundation No.7182182 to R. Y; International Science \& Technology Cooperation Program of China (2015DFB30040 to S.S. and Y.Z.) and the National Science and Technology Major Project of the Ministry of Science and Technology of China No. 2018ZX10302207.

\section{Author Contributions}

YZ, RY, LZ contributed to conception and design, collection and assembly of data, data analysis and interpretation, and final approval of the manuscript. HH and SC, TZ contributed to collection data and interpretation, and final approval of the manuscript. YZ contributed to critically editing the manuscript, and final approval of the manuscript.

\section{Ethics approval and consent to participate}

This study was approved by the Animal Care and Use Committee of the Health Science Center, Peking University (\# 2015-186).

\section{Consent for publication}

No applicable

\section{Competing interests}

The authors declare that they have no competing interests

\section{References}


1. Tansel A, Katz LH, El-Serag HB, Thrift AP, Parepally M, Shakhatreh MH, et al. Incidence and Determinants of Hepatocellular Carcinoma in Autoimmune Hepatitis: A Systematic Review and Metaanalysis. Clin Gastroenterol Hepatol. 2017;15:1207-1217.e4.

2. Gatselis NK, Zachou K, Koukoulis GK, Dalekos GN. Autoimmune hepatitis, one disease with many faces: etiopathogenetic, clinico-laboratory and histological characteristics. World J Gastroenterol. 2015;21:60-83.

3. Sahebjam F, Vierling JM. Autoimmune hepatitis. Front Med. 2015;9:187-219.

4. Czaja AJ. Diagnosis and Management of Autoimmune Hepatitis: Current Status and Future Directions. Gut Liver. 2016;10:177-203.

5. Czaja AJ, Bianchi FB, Carpenter HA, Krawitt EL, Lohse AW, Manns MP, et al. Treatment challenges and investigational opportunities in autoimmune hepatitis. Hepatology. 2005;41:207-15.

6. Squillaro T, Peluso G, Galderisi U. Clinical Trials With Mesenchymal Stem Cells: An Update. Cell Transplant. 2016;25:829-48.

7. Zhu X, He B, Zhou X, Ren J. Effects of transplanted bone-marrow-derived mesenchymal stem cells in animal models of acute hepatitis. Cell Tissue Res. 2013;351:477-86.

8. Higashimoto M, Sakai Y, Takamura M, Usui S, Nasti A, Yoshida K, et al. Adipose tissue derived stromal stem cell therapy in murine ConA-derived hepatitis is dependent on myeloid-lineage and CD4+ T-cell suppression. Eur J Immunol. 2013;43:2956-68.

9. Ryu K-H, Kim S-Y, Kim Y-R, Woo S-Y, Sung SH, Kim HS, et al. Tonsil-derived mesenchymal stem cells alleviate concanavalin A-induced acute liver injury. Exp Cell Res. 2014;326:143-54.

10. Miura M, Gronthos S, Zhao M, Lu B, Fisher LW, Robey PG, et al. SHED: stem cells from human exfoliated deciduous teeth. Proc Natl Acad Sci USA. 2003;100:5807-12.

11. Nakamura S, Yamada Y, Katagiri W, Sugito T, Ito K, Ueda M. Stem cell proliferation pathways comparison between human exfoliated deciduous teeth and dental pulp stem cells by gene expression profile from promising dental pulp. J Endod. 2009;35:1536-42.

12. Yamaza T, Alatas FS, Yuniartha R, Yamaza H, Fujiyoshi JK, Yanagi Y, et al. In vivo hepatogenic capacity and therapeutic potential of stem cells from human exfoliated deciduous teeth in liver fibrosis in mice. Stem Cell Res Ther. 2015;6:171.

13. Ohkoshi $S$, Hirono H, Nakahara T, Ishikawa H. Dental pulp cell bank as a possible future source of individual hepatocytes. World J Hepatol. 2018;10:702-7.

14. Bies C, Lehr C-M, Woodley JF. Lectin-mediated drug targeting: history and applications. Adv Drug Deliv Rev. 2004;56:425-35.

15. Heymann F, Hamesch K, Weiskirchen R, Tacke F. The concanavalin A model of acute hepatitis in mice. Lab Anim. 2015;49:12-20.

16. Erhardt A, Biburger M, Papadopoulos T, Tiegs G. IL-10, regulatory T cells, and Kupffer cells mediate tolerance in concanavalin A-induced liver injury in mice. Hepatology. 2007;45:475-85. 
17. Wang L, Tu L, Zhang J, Xu K, Qian W. Stellate Cell Activation and Imbalanced Expression of TGF$\beta 1 /$ TGF- $\beta 3$ in Acute Autoimmune Liver Lesions Induced by ConA in Mice. Biomed Res Int. 2017;2017:2540540.

18. Schümann J, Wolf D, Pahl A, Brune K, Papadopoulos T, van Rooijen N, et al. Importance of Kupffer cells for T-cell-dependent liver injury in mice. Am J Pathol. 2000;157:1671-83.

19. Li J, Chen K, Li S, Liu T, Wang F, Xia Y, et al. Pretreatment with Fucoidan from Fucus vesiculosus Protected against ConA-Induced Acute Liver Injury by Inhibiting Both Intrinsic and Extrinsic Apoptosis. PLoS ONE. 2016;11:e0152570.

20. Schwabe RF, Brenner DA. Mechanisms of Liver Injury. I. TNF-alpha-induced liver injury: role of IKK, JNK, and ROS pathways. Am J Physiol Gastrointest Liver Physiol. 2006;290:G583-589.

21. Li J, Wang F, Xia Y, Dai W, Chen K, Li S, et al. Astaxanthin Pretreatment Attenuates Hepatic Ischemia Reperfusion-Induced Apoptosis and Autophagy via the ROS/MAPK Pathway in Mice. Mar Drugs. 2015;13:3368-87.

22. Martinez Saez D, Sasaki RT, Neves A da C, da Silva MCP. Stem Cells from Human Exfoliated Deciduous Teeth: A Growing Literature. Cells Tissues Organs (Print). 2016;202:269-80.

23. Mohan RR, Mohan RR, Kim WJ, Wilson SE. Modulation of TNF-alpha-induced apoptosis in corneal fibroblasts by transcription factor NF-kappaB. Invest Ophthalmol Vis Sci. 2000;41:1327-36.

24. Balitzer D, Shafizadeh N, Peters MG, Ferrell LD, Alshak N, Kakar S. Autoimmune hepatitis: review of histologic features included in the simplified criteria proposed by the international autoimmune hepatitis group and proposal for new histologic criteria. Mod Pathol. 2017;30:773-83.

25. Xie F, He J, Chen Y, Hu Z, Qin M, Hui T. Multi-lineage differentiation and clinical application of stem cells from exfoliated deciduous teeth. Hum Cell. 2020;

26. Constant SL, Bottomly K. Induction of Th1 and Th2 CD4+ T cell responses: the alternative approaches. Annu Rev Immunol. 1997;15:297-322.

27. Wolf D, Hallmann R, Sass G, Sixt M, Küsters S, Fregien B, et al. TNF-alpha-induced expression of adhesion molecules in the liver is under the control of TNFR1-relevance for concanavalin A-induced hepatitis. J Immunol. 2001;166:1300-7.

28. Ksontini R, Colagiovanni DB, Josephs MD, Edwards CK, Tannahill CL, Solorzano CC, et al. Disparate roles for TNF-alpha and Fas ligand in concanavalin A-induced hepatitis. J Immunol. 1998;160:40829.

29. Otabe K, Muneta T, Kawashima N, Suda H, Tsuji K, Sekiya I. Comparison of Gingiva, Dental Pulp, and Periodontal Ligament Cells From the Standpoint of Mesenchymal Stem Cell Properties. Cell Med. 2012;4:13-21.

30. Li J, Xia Y, Liu T, Wang J, Dai W, Wang F, et al. Protective effects of astaxanthin on ConA-induced autoimmune hepatitis by the JNK/p-JNK pathway-mediated inhibition of autophagy and apoptosis. PLOS ONE. 2015;10:e0120440.

31. Tao Y, Yan X, Zhou T, Shen L, Liu Z, Liu C. Fuzheng Huayu recipe alleviates hepatic fibrosis via inhibiting TNF-a induced hepatocyte apoptosis. BMC Complement Altern Med. 2014;14:449. 
32. Zhang P, Yin Y, Wang T, Li W, Li C, Zeng X, et al. Maresin 1 mitigates concanavalin A-induced acute liver injury in mice by inhibiting ROS-mediated activation of NF-KB signaling. Free Radic Biol Med. 2020;147:23-36.

33. Chen Y-Y, Li R-Y, Shi M-J, Zhao Y-X, Yan Y, Xu X-X, et al. Demethyleneberberine alleviates inflammatory bowel disease in mice through regulating NF-KB signaling and T-helper cell homeostasis. Inflamm Res. 2017;66:187-96.

34. Mehmood T, Maryam A, Zhang H, Li Y, Khan M, Ma T. Deoxyelephantopin induces apoptosis in HepG2 cells via oxidative stress, NF-KB inhibition and mitochondrial dysfunction. Biofactors. 2017;43:63-72.

35. ELnahas EM, Zeid MSA, Kawy HSA, Hendawy N, Baher W. Celecoxib attenuates depressive-like behavior associated with immunological liver injury in C57BL/ 6 mice through TNF- $a$ and NF-kb dependent mechanisms. Life Sci. 2016;163:23-37.

36. Liu T, Xia Y, Li J, Li S, Feng J, Wu L, et al. Shikonin Attenuates Concanavalin A-Induced Acute Liver Injury in Mice via Inhibition of the JNK Pathway. Mediators Inflamm. 2016;2016:2748367.

37. Mao Y, Wang J, Yu F, Cheng J, Li H, Guo C, et al. Ghrelin reduces liver impairment in a model of concanavalin A-induced acute hepatitis in mice. Drug Des Devel Ther. 2015;9:5385-96.

\section{Figures}


A

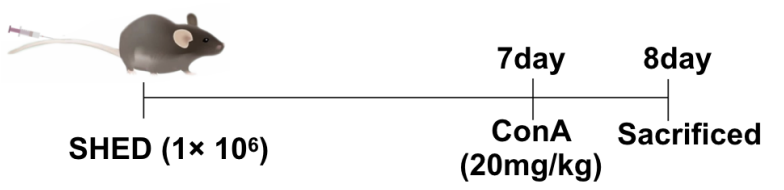

B

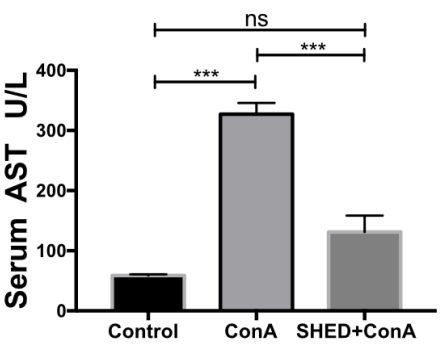

C

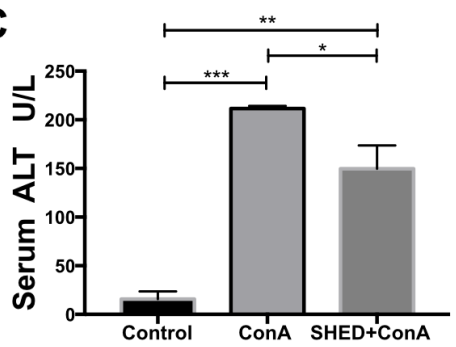

D

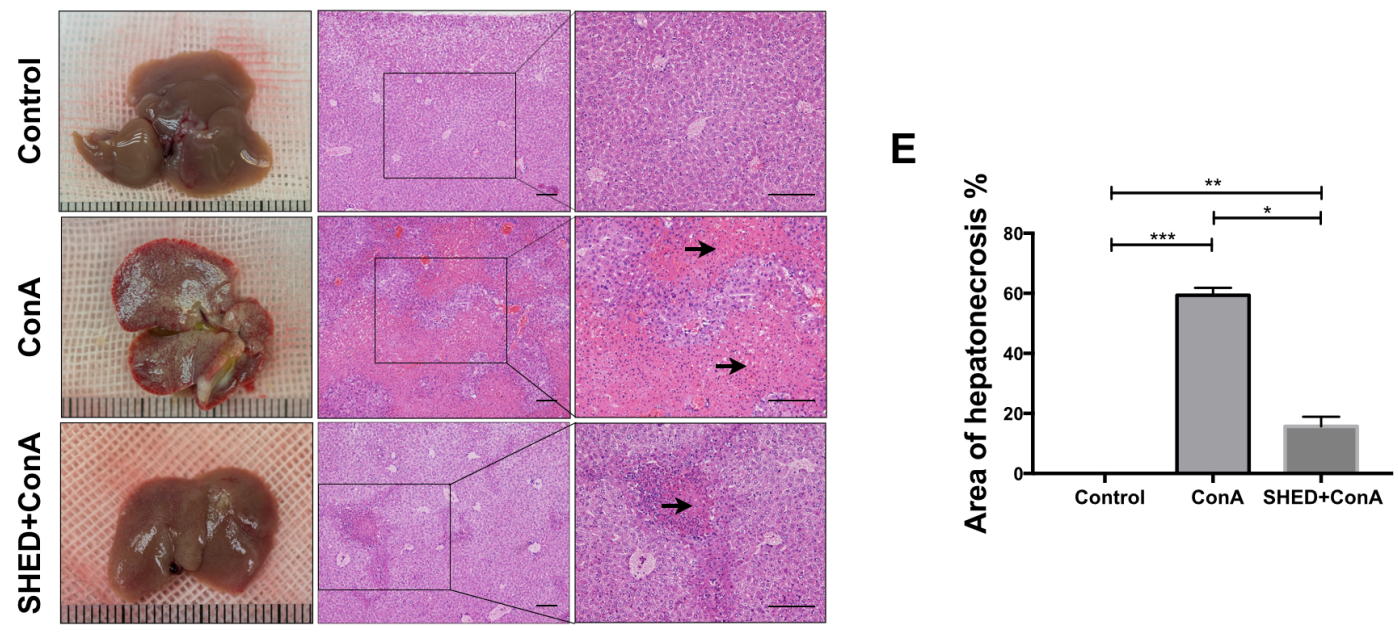

Figure 1

\section{Figure 1}

SHED-pretreatment moderated ConA-induced acute liver injury in mice. a Mice were subjected to intravenous injections with SHED cell $(1 \times 106) 7 \mathrm{~d}$ prior with Con A $(20 \mathrm{mg} / \mathrm{kg})$ challenge. After injection for 1d, mice were scarified. b The level of serum ALT. c The level of serum AST. $d$ Anatomical examinations and $\mathrm{HE}$ staining showed necrotic area in three groups. The arrows indicate massive cell death in the liver section. $e$ The percentage of liver tissue necrotic areas. scale $=200 \mu \mathrm{M}(\mathrm{d}), * * *, \mathrm{P}<0.001, * *, \mathrm{P}<0.01, *$, $P<0.05$, ns showed no statistical difference (mean $\pm S D), n \geq 3(b, c, e)$. 

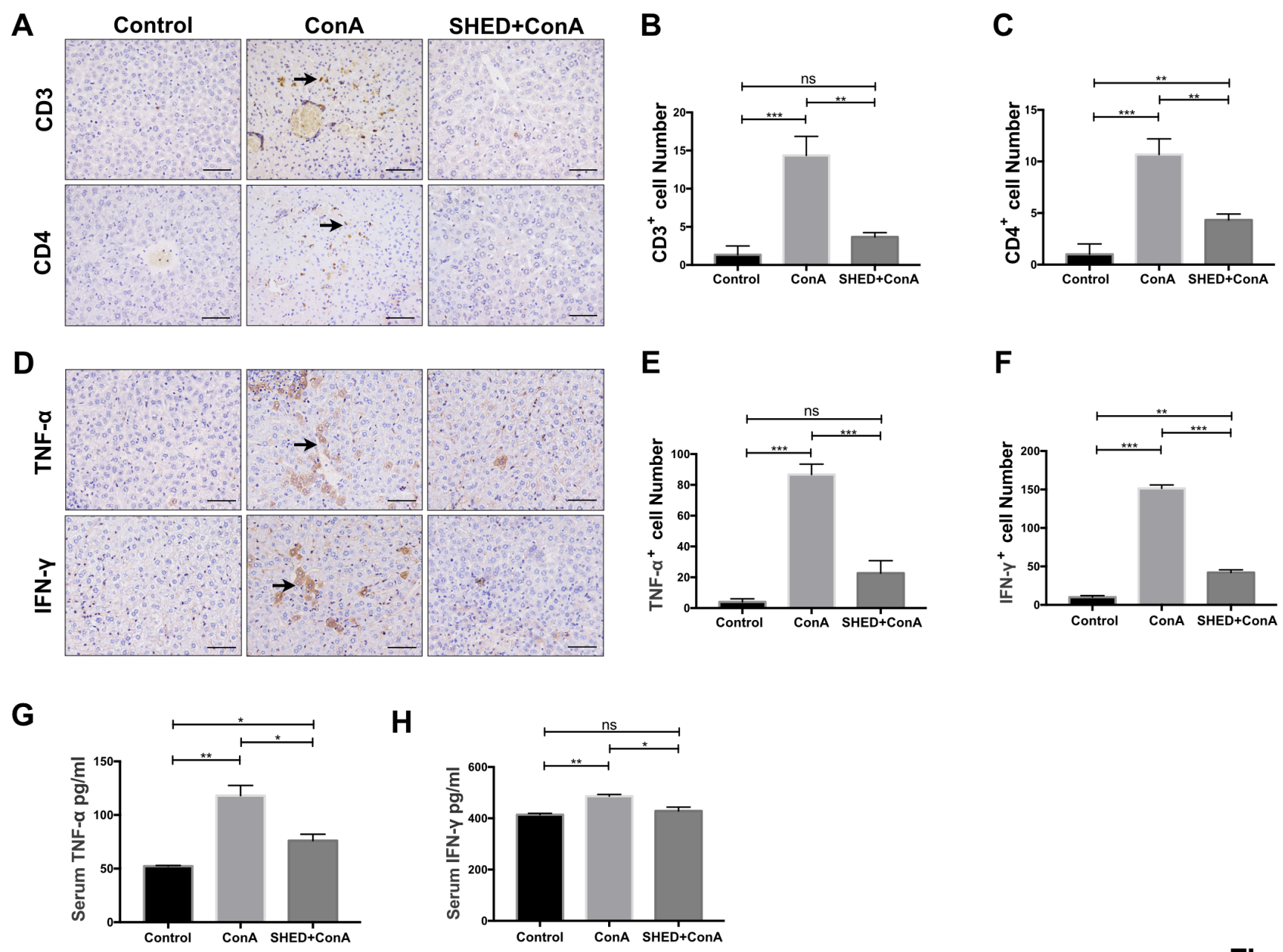

Figure 2

Figure 2

SHED-pretreatment reduced inflammation in ConA-induced hepatitis. a The expression of CD3+, CD4+ T cells in the liver tissues. $b$ Quantitative analysis showed the number of CD3+ T cells. c Quantitative analysis showed the number of CD4+ T cells. $d$ The expression of TNF- $a$ and IFN- $y$ in the liver tissues. e Quantitative analysis showed that the number of TNF-a+ cell. $f$ Quantitative analysis showed that the number of IFN- $-\mathrm{+}$ cell. $g$ The level of serum TNF- $a$. h The level of serum IFN- - . The arrows indicate positive cell in the liver section. scale $=100 \mu \mathrm{M}(\mathrm{a}, \mathrm{d}), * * *, P<0.001, * *, P<0.01, *, P<0.05$, ns showed no statistical difference (mean $\pm S D), n \geq 3(b, c, e, f, g, h)$. 

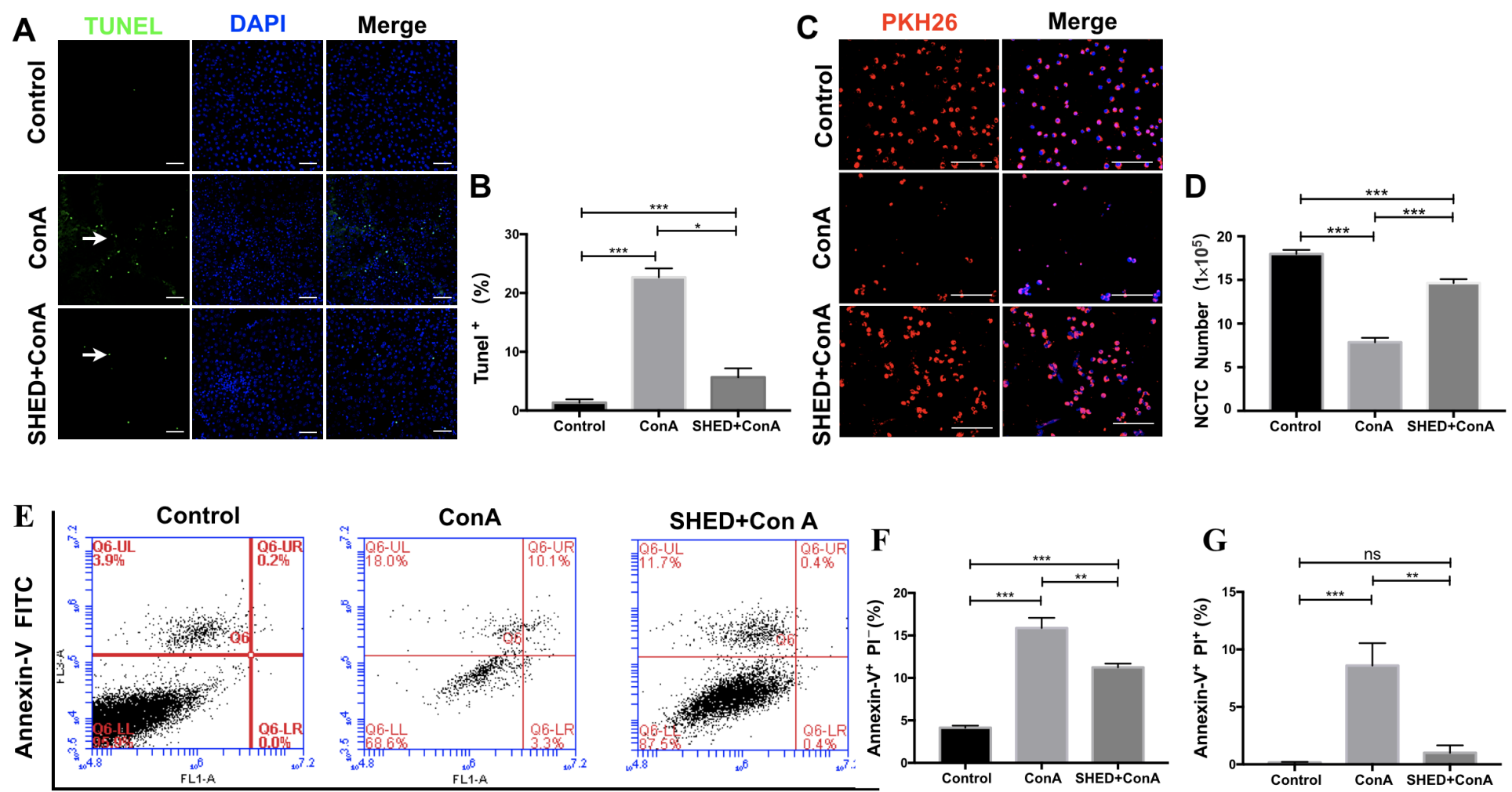

PI

Figure 3

\section{Figure 3}

SHED-pretreatment inhibited apoptosis of hepatocytes induced by Con A. a TUNEL staining showed apoptotic cells. b Semi-quantitative analysis showed the percent of Tunel+ area in liver tissues. The arrows indicate positive cell death in the liver section. c NCTC-1469 liver cell number in the indicated treatment group, NCTC cells was labeled by PKH26. d Quantitative analysis showed NCTC-1469 liver cell number. e Apoptosis of NCTC cells were detected by flow cytometry. $f$ Quantitative analysis of Annexin $\mathrm{V}+\mathrm{PI}$ - rate. g Quantitative analysis of Annexin V+PI+ rate. scale $=100 \mu \mathrm{M}(\mathrm{a}, \mathrm{c}), * \star \star *, \mathrm{P}<0.001, * *, \mathrm{P}<$ $0.01, *, P<0.05$, ns showed no statistical difference (mean $\pm S D), n \geq 3(b, d, f)$. 
A

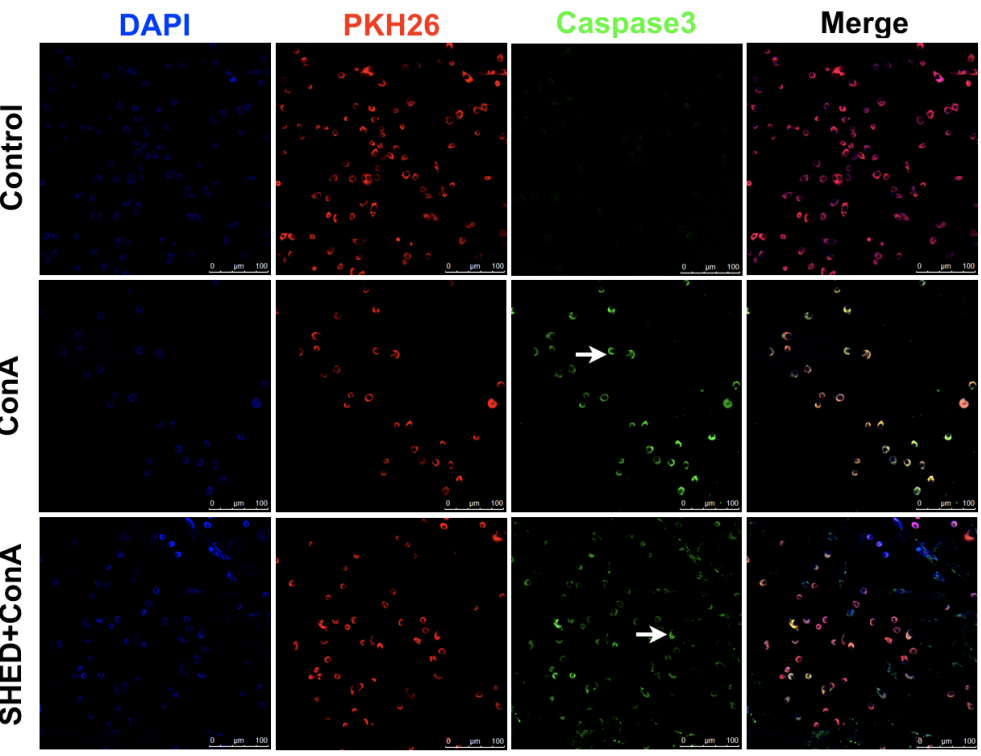

C

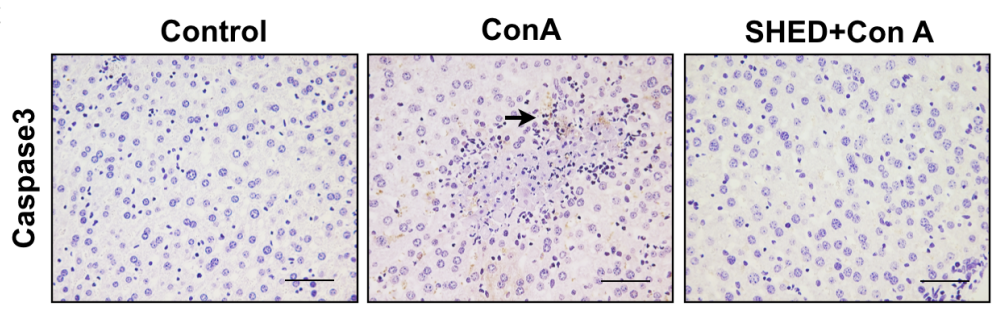

B

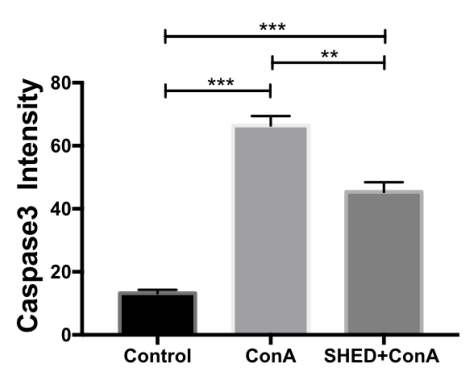

D

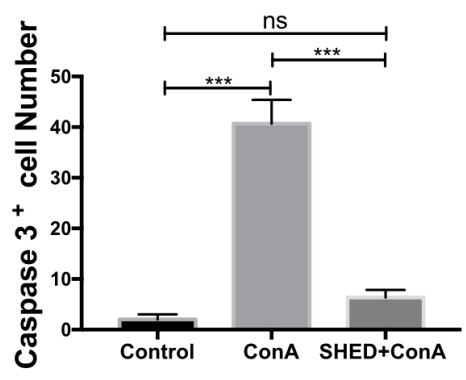

Figure 4

\section{Figure 4}

SHED-pretreatment reduced caspase 3 in ConA-induced hepatitis. a The expression of caspase3 protein in NCTC cells. b Semi quantitative analysis showed that the fluorescence intensity of caspase3 protein. c The expression of caspase3 protein in liver tissues. $d$ Quantitative analysis showed the number of caspase $3+$ cell. The arrows indicate positive cell in the liver section. scale $=100 \mu \mathrm{M}(\mathrm{a}, \mathrm{c}), * \star \star, \mathrm{P}<0.001$, $* *, P<0.01, *, P<0.05$, ns showed no statistical difference (mean $\pm S D), n \geq 3(b, d)$. 

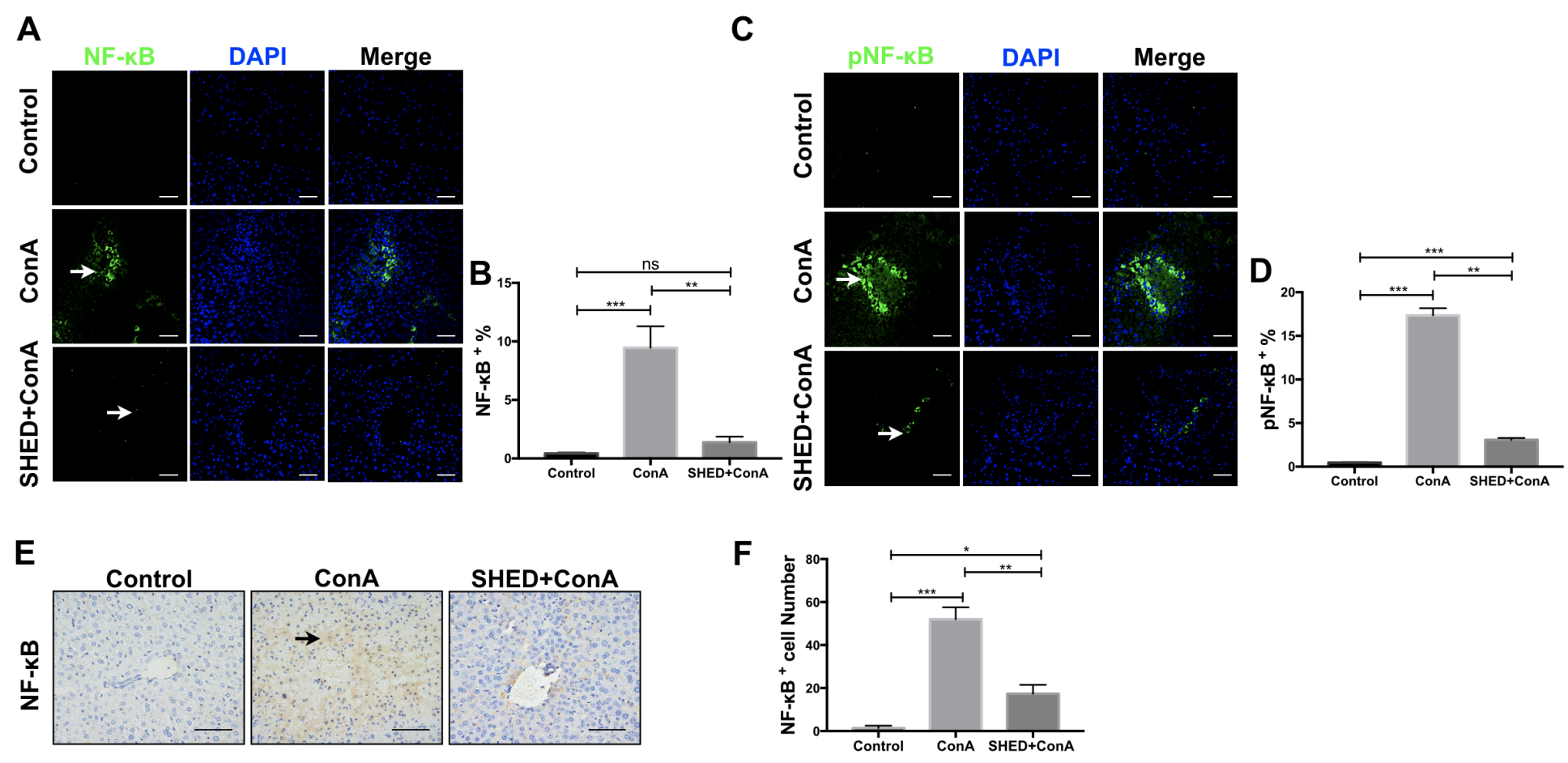

Figure 5

\section{Figure 5}

SHED-pretreatment reduced NF-kB in ConA-induced hepatitis. a The expression of NF-kB protein in liver tissues. $b$ Quantitative analysis showed the number of NF-KB + cells of protein. $c$ The expression of pNF$\kappa B$ protein in liver tissues. $d$ Quantitative analysis showed the number of pNF-kB+cell. e Immunohistochemistry showed the expression of NF-KB protein in liver tissues $f$ Quantitative analysis showed the number of NF-kB+ cells of protein in Immunohistochemistry. The arrows indicate positive cell in the liver section. scale $=100 \mu \mathrm{M}(\mathrm{a}, \mathrm{c}, \mathrm{e}), * * *, \mathrm{P}<0.001, * *, \mathrm{P}<0.01, *, \mathrm{P}<0.05$, ns showed no statistical difference (mean $\pm S D), n \geq 3(b, d, f)$. 


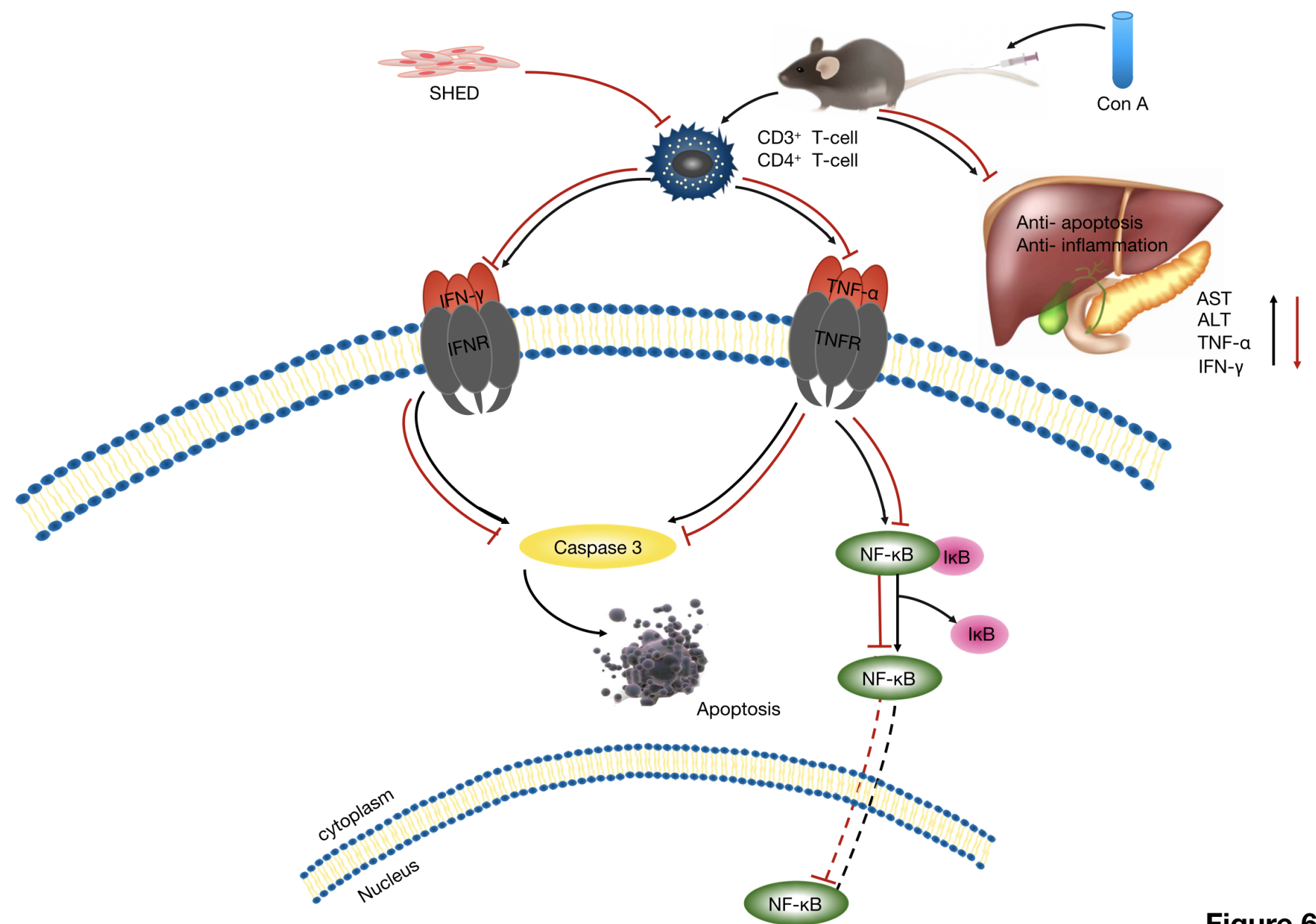

Figure 6

Figure 6

Schema of SHED treatment for ConA-induced hepatitis. In ConA-induced acute liver injury, TNF-a and IFN- $\gamma$ activated the NF-KB and caspase3 to induce hepatocyte apoptosis. SHED alleviated ConA-induced acute liver injury via inhibition of hepatocyte apoptosis mediated by the NF-KB pathways.

\section{Supplementary Files}

This is a list of supplementary files associated with this preprint. Click to download.

- supplementaryfigure1.pdf 